\title{
Identification and quality assessment of commercial valerian samples using DNA barcoding and UHPLC-MS
}

\author{
Marta Sánchez, Elena González-Burgos, M. Pilar Gómez-Serranillos \\ Department of Pharmacology, Pharmacognosy and Botany, Faculty of Pharmacy, Universidad Complutense de \\ Madrid, Plaza Ramon y Cajal s/n, Ciudad Universitaria, 28040, Madrid, Spain
}

\begin{abstract}
Introduction
The root of $V$. officinalis $L$, has been widely used for sleep troubles, anxiety and states of nervousness. The main compound responsible for these activities is the states of nervousness. The main compound responsible for these activities is the
sesquiterpene valerenic acid which acts by modulating GABA receptors (Felgentreff et al., 2012). Apart from valerenic acid, there have been identified a great diversity of bioactive compounds in Valeriana officinalis including iridoids such as valepotriate and isovalepotriate, flavonoids such as luteolin and quercetin, lignanoids such as $8^{\prime}$-hydroxypinoresinol, alkaloids such as valerine and valerianine and other terpenes such as isovaleric acid, valeric acid and acetoxyvalerenic (Patpcka \& Jakl, 2010; Wang et al., 2010; Chen et al., 2013; Wang et al., 2013; Chen et al., 2015).

Over the past two decades, there have been an increase in medicinal plants consumption for the treatment of minor syndromes (Shaw et al., 2012). Medicinal plants must comply with quality, safety and efficacy standards. DNA barcoding is an effective tool to ensure correct identification and authentication of medicinal plants. This technique consists of using short DNA sequences from standardized genetic regions (rbcL, matK and ITS2) (Group CPW, 2009; Pawar et al., 2017). Moreover, Ultra High Performance Liquid Chromatography coupled with Mass Spectrometry (UHPLC-MS) is a very useful technique to identify and quantify bioactive compounds presented in medicinal plants.
\end{abstract}

\section{Objectives}

To apply the DNA-Barcoding technique and the UHPLC-MS methodology as tools to evaluate the quality of commercial samples of Valeriana officinalis $\mathrm{L}$.

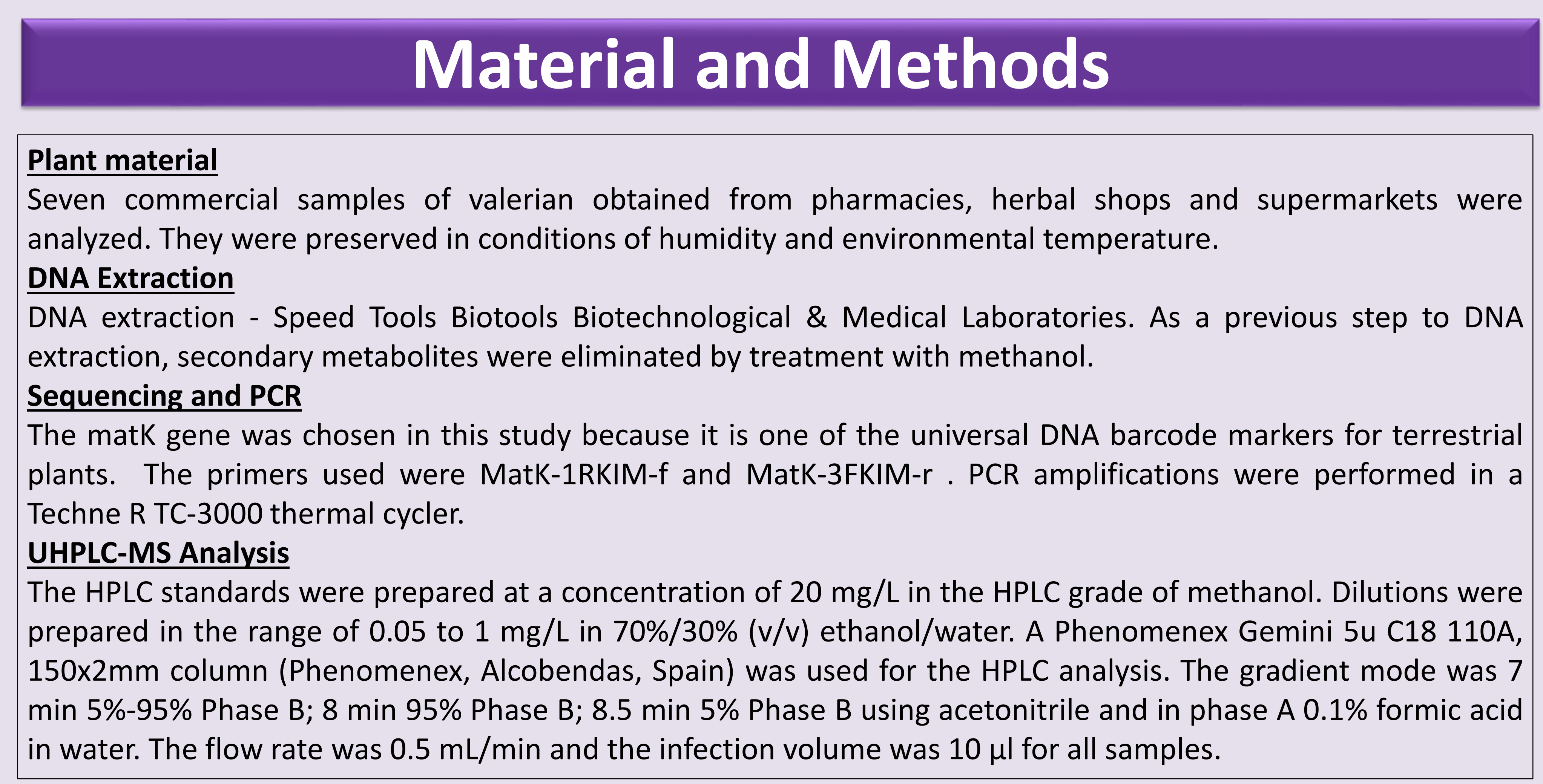

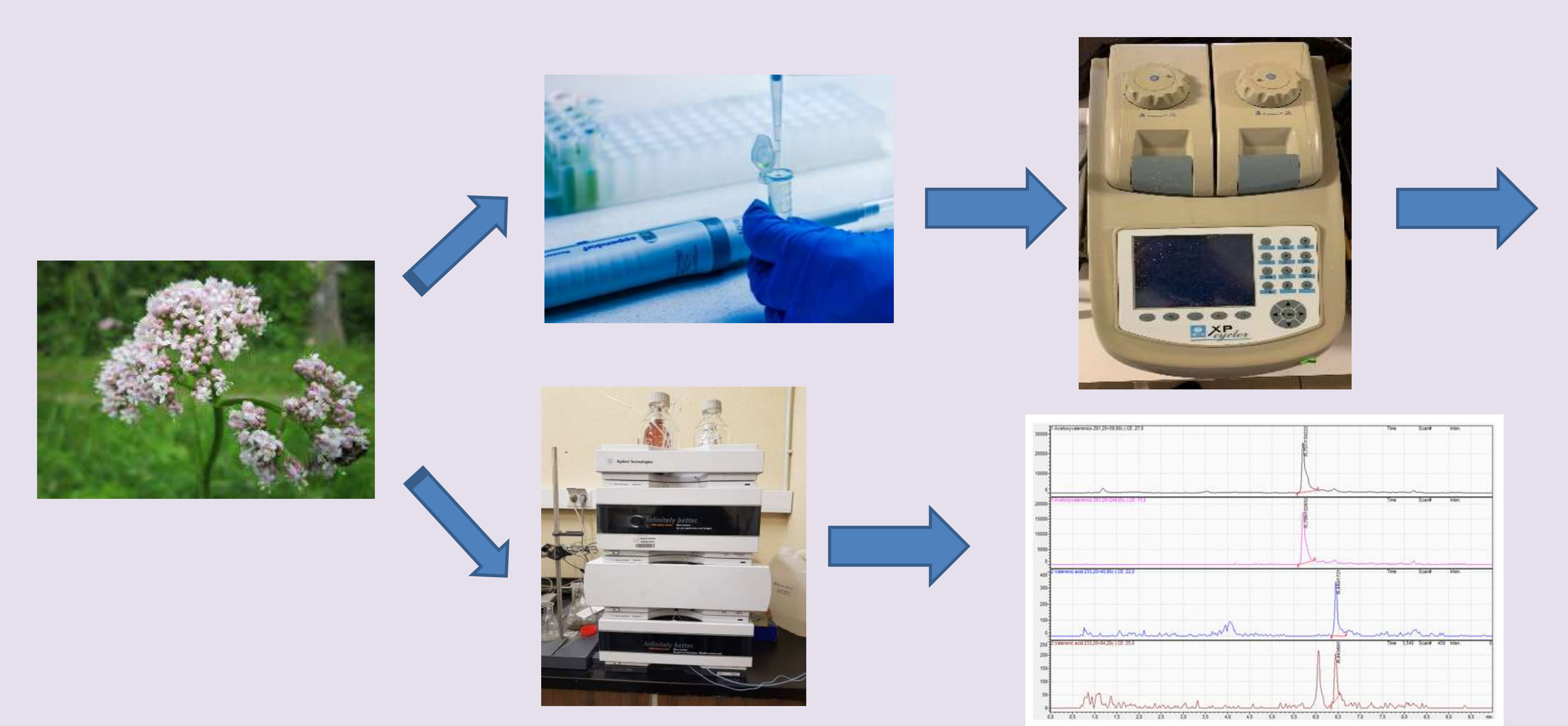

\section{Results and discusion}

\section{DNA-BARCODING ANALYSIS}

\section{Sequencing}

The DNA sequences were manually assembled and adjusted through the BioEdit sequence alignment editor software ( $v$ 7.2). A second edition and assembly of the sequence fragments was done with the SeqMan v.7 program (Lasergene R, DNASTAR, Madison, Wisconsin, USA). The sequence identity was evaluated using the mega-BLAST search function in GenBank. Each data set was aligned using MAFFT v.7 implementing the GINS-I alignment algorithm, score matrix ' $1 \mathrm{PAM} / \mathrm{K}=2$ ' with a compensation value of 0.0 , and the rest of the parameters set to default values. The identification of the samples was evaluated using the Life Barcode Data System (BOLD Systems v3).

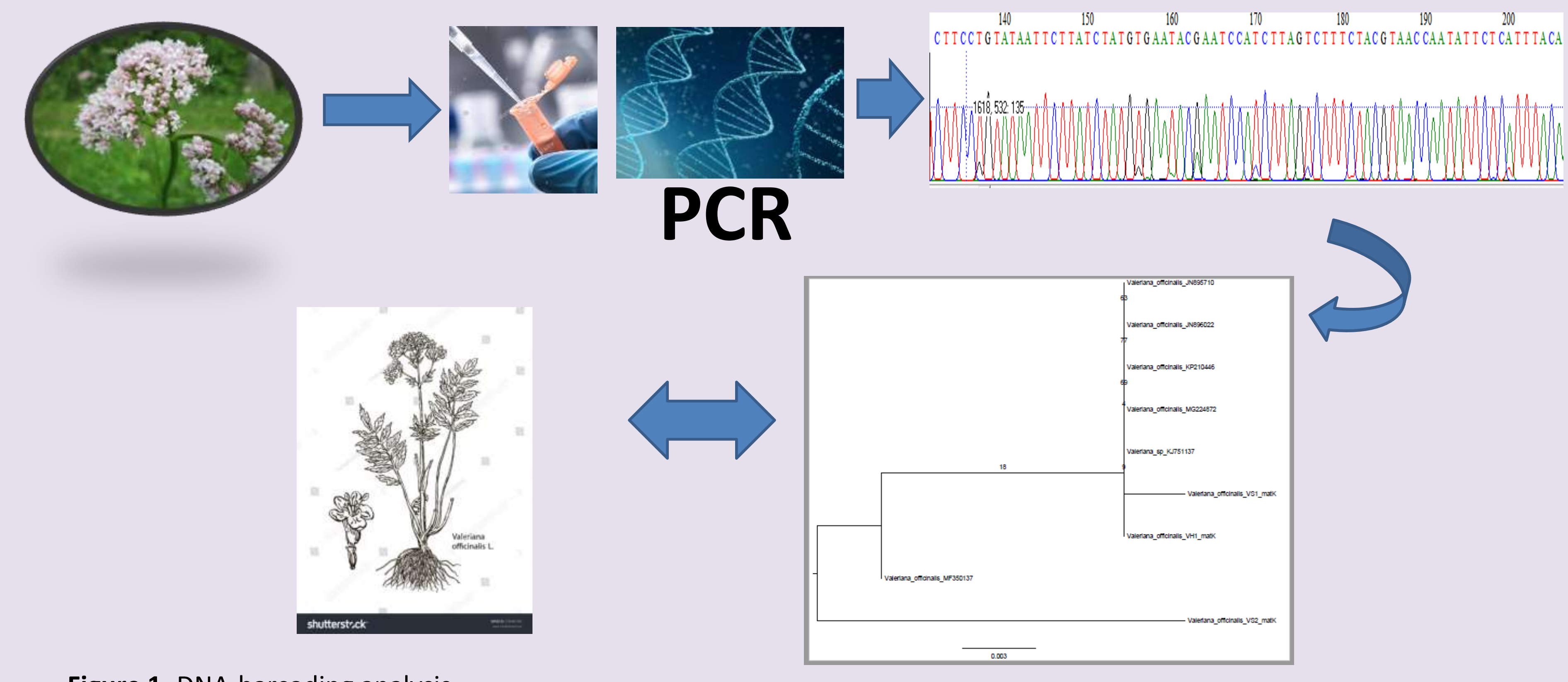

Figure 1. DNA-barcoding analysis.

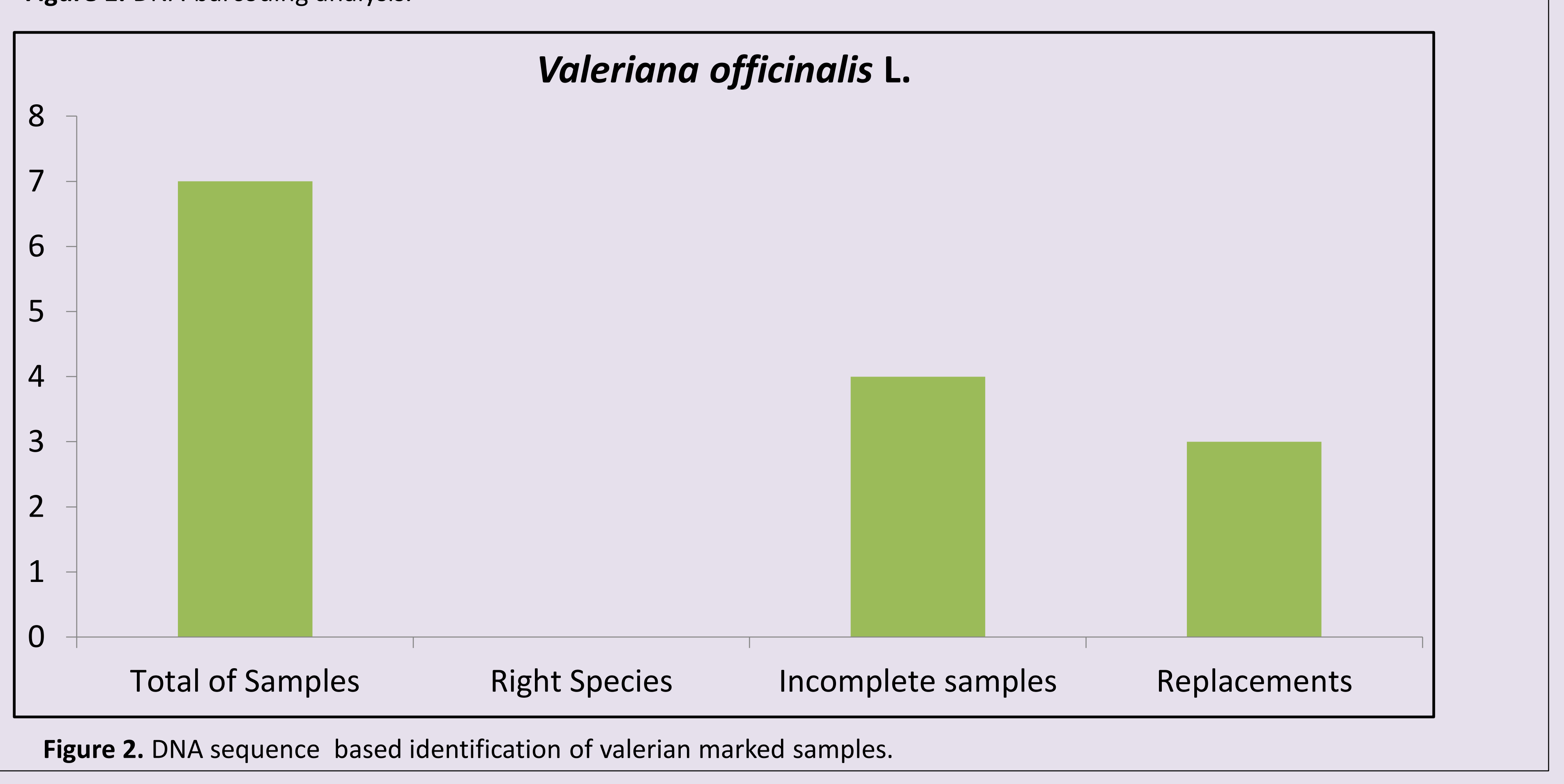

UHPLC-MS

The compounds valerenic acid and acetoxy valerenic acid were identified in all study samples.

\begin{tabular}{|c|c|c|c|}
\hline Sample & $\begin{array}{l}\text { Acetoxyvalerenic acid } \\
\text { (\%) }\end{array}$ & $\begin{array}{l}\text { Valerenic acid } \\
(\%)\end{array}$ & \\
\hline VH1 & 0.026 & 0.109 & - \\
\hline VH2 & 0.024 & 0.115 & \\
\hline $\mathrm{VH3}$ & 0.024 & 0.101 & \\
\hline VF1 & 0.020 & 0.048 & \\
\hline VF2 & 0.029 & 0.078 & 11 \\
\hline VF3 & 0.053 & 0.167 & \\
\hline vS1 & 0.025 & 0.084 & Figure 3. Representative UHPLC-ESI-QqQ- \\
\hline
\end{tabular}

\section{Conclusions}

- DNA was extracted from 3 of the 7 analyzed samples of Valeriana officinalis

- These 3 valerian samples were labelled as Valeriana officinalis but they were identified as Valeriana hirtella Kunth

- DNA-barcoding is an effective method to guarantee the quality control of plant species.

- UHPL-MS revealed that acetoxy valerenic acid concentration was from $0.020 \%$ to $0.053 \%$ whereas valerenic acid content was from $0.048 \%$ to $0.167 \%$.

\section{References}

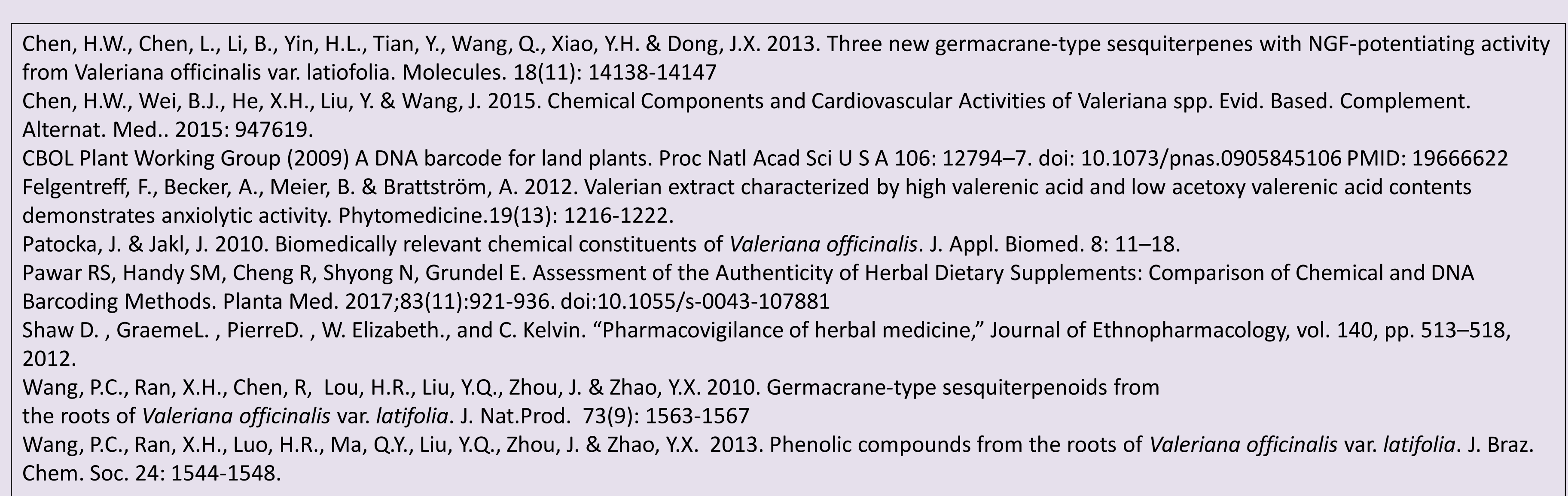

